\title{
Microvariability of Line Profiles in the Spectra of OB Stars: III. The Supergiant $\rho$ LEO
}

\author{
A. F. Kholtygin ${ }^{1}$, S. N. Fabrika ${ }^{2}$, T. E. Burlakova ${ }^{2,3}$, G. G. Valyavin ${ }^{2,3}$, G. A. Chuntonov ${ }^{2}$, \\ D. O. Kudryavtsev², D. Kang ${ }^{3}$, M. V. Yushkin ${ }^{2}$, and G. A. Galazutdinov ${ }^{2,4}$ \\ ${ }^{I}$ Sobolev Astronomical Institute, St. Petersburg State University, \\ Bibliotechnaya pl. 2, Petrodvorets, 198904 Russia \\ ${ }^{2}$ Special Astrophysical Observatory, Russian Academy of Sciences, \\ Nizhniŭ Arkhyz, Karachă̌-Cherkessian Republic, 357147 Russia \\ ${ }^{3}$ Bohyunsan Optical Astronomy Observatory, Jaceon P.O.B., \\ Young-chun, Kyung-pook, 770-820, South Korea \\ ${ }^{4}$ Korea Astronomy and Space Science Institute, Daejeon, Korea \\ Received December 16, 2006; in final form, April 5, 2007
}

\begin{abstract}
We observed the bright supergiant $\rho$ Leo (B1 Iab) in January-February 2004 using the 6-m telescope of the Special Astrophysical Observatory (Russia) and the 1.8-m telescope of the Bohyunsan Optical Astronomy Observatory (South Korea). 47 spectra with high time resolution (4-10 min), signalto-noise ratios 300-1000, and spectral resolutions 45000-60 000 were obtained. We detected variability in the HeI, SiII, SiIII, and NII line profiles, which may be due to rotational modulation of the profiles and photospheric pulsations of $\rho$ Leo. The possible influence of the stellar magnetic field on the line-profile variations is discussed.
\end{abstract}

PACS numbers: 97.20.Pm, 95.85.Kr, 97.10.Sj

DOI: $10.1134 / \mathrm{S} 1063772907110054$

\section{INTRODUCTION}

Spectral observations of hot stars at UV [1,2], optical $[1-6]$ and $\mathrm{X}$-ray $[7,8]$ wavelengths indicate the presence of structures of various sizes and densities with lifetimes from fractions of an hour to several days in the atmospheres of these stars. Variations of the line profiles in the spectra of OB stars are primarily regular or quasi-regular. In the spectra of numerous $\mathrm{B}$ stars and six $\mathrm{O}$ stars, regular short-period (3-12 h) variations of the profiles of HeI, SiIII, and other ions related to non-radial pulsations (NRPs) have been detected [6].

In contrast to B stars, the amplitudes of the lineprofile variations of $\mathrm{O}$ stars and stars of early $\mathrm{B}$ subtypes $(\mathrm{B} 1-\mathrm{B} 3)$ are relatively small $(1-3 \%$; see, for example, [9]). In other words, the line profiles in these stars display microvariability. The detection and, especially, clarification of the nature of this variability requires observations with high time and spectral resolution and with signal-to-noise ratios $\geq 300$.

The magnetic fields (several hundred $\mathrm{G}$ at the stellar surface) may also facilitate the formation of largescale structures in the atmospheres of OB stars [10]. Currently, magnetic fields have been detected confidently for only two $O$ stars $\left(\theta^{1}\right.$ Ori C [11] and
HD 191612 [12]), as well as for a number of earlysub-type B stars [13].

We suggested a program of variability studies and searches for evidence for the presence of magnetic fields in late-O and early-B stars [14], which extends our previous studies of line-profile microvariability in the spectra of hot stars $[9,15,16]$.

Here, we describe observations of the BlIab supergiant $\rho$ Leo carried out in 2004 and present the results of studies of line-profile microvariability in the spectrum of this star. In 2005-2006, we observed this star using a circular-polarization analyzer mounted on the 6-m telescope of the Special Astrophysical Observatory (SAO); these observations, together with the results of our search for a magnetic field in $\rho$ Leo, will be described in a future paper.

Section 2 presents the basic data for the star and describes our observing and spectral-reduction methods. The results of our search for regular lineprofile variations are presented in Section 3. Section 4 discusses the evolutionary status of the star and the influence of its magnetic field on the variability of the line profiles. In the final section, we formulate the conclusions of our study. 
2. BASIC PARAMETERS OF THE STAR. OBSERVATIONS AND REDUCTION OF THE SPECTRA

The supergiant $\rho$ Leo (HD 91316) is a relatively slowly rotating B1 Iab star. Its effective temperature $T_{e f f}$ is somewhat uncertain, with values of $T_{e f f}=$ $20260 \mathrm{~K}$ [17], $T_{\text {eff }}=24200 \mathrm{~K}$ [18], and $T_{\text {eff }}=$ $22000 \mathrm{~K}$ [19] being given in various studies.

In the Hertzsprung-Russell (HR) diagram, $\rho$ Leo is situated in the region of early-B $\beta$ Cep variables (see, for example, [20]). Table 1 presents the parameters of the star: $T_{\text {eff }}$ is its effective temperature, $M$ the mass of the primary component of the system, $\dot{M}$ the mass-loss rate of the star, $L$ the bolometric luminosity, $V_{\infty}$ the terminal velocity of the stellar wind, $V \sin i$ the rotational velocity of the star, and $\log \varepsilon(\mathrm{El})$ elemental abundances on a $\log \varepsilon(\mathrm{H})=12$ scale.

In 2004, we observed $\rho$ Leo using the 6 -m telescope of the SAO and the 1.8-m telescope of the Bohyunsan Optical Astronomy Observatory (BOAO, South Korea). Table 2 presents information about the observations. The SAO spectral observations on January 10-11, 2004 were carried out at $\lambda \lambda$ 4500-6000 $\AA$ using the NES quartz echelle spectrograph [21], which is permanently mounted at the Nasmyth focus and outfitted with a $2048 \times 2048$ CCD detector (Uppsala CCD).

An image cutter was used to divide the image into three slices in order to increase the limiting magnitude of the spectrograph [22]. In this observing mode, the spectral resolution is $R \approx 60000$ and the dispersion $0.033 \AA$ /pixel. The image size (seeing) was about $3^{\prime \prime}$. The comparison spectrum was provided by a Tho-Ar lamp. When the image cutter was applied, each echelle order of the image was represented by three sub-orders (cuts). The instrumental shift of the upper and lower cuts in an individual order relative to the middle cut was determined by the cross correlation of the images of the comparison spectrum. The three cuts obtained in an individual order were summed using the median average method, taking into account the obtained instrument shifts.

The preliminary reduction of the CCD images of the echelle spectra was carried out in MIDAS package [9]. We adapted the standard ECHELLE procedure for the data obtained with the image cutter. The processing included median filtering and averaging of the underlying background (bias), with its subsequent subtraction from the remaining frames, and removal of cosmic-ray traces. The positions of the spectral orders were determined using the method of Ballester [25].

To determine the contribution of scattered light, the inter-order space was identified on the frames and
Table 1. The parameters of $\rho$ Leo system

\begin{tabular}{l|c|c}
\hline \multicolumn{1}{c|}{ Parameter } & Value & Reference \\
\hline$T_{\text {eff }}, \mathrm{K}$ & 24200 & {$[18]$} \\
$\log g$ & 3.09 & {$[18]$} \\
$M / M_{\odot}$ & 22 & {$[17]$} \\
$R / R_{\odot}$ & 32 & {$[17]$} \\
& 37.4 & {$[19]$} \\
$V_{\infty}, \mathrm{km} / \mathrm{s}$ & 1110 & {$[19,23]$} \\
$-\log \left(\dot{M} / M_{\odot}\right)$ & -6.20 & {$[17]$} \\
$\log \left(L / L_{\odot}\right)$ & 5.18 & {$[17]$} \\
$V \sin i, \mathrm{~km} / \mathrm{s}$ & 75 & {$[19,23]$} \\
& 60 & {$[24]$} \\
$\log \varepsilon(\mathrm{C})$ & 7.5 & {$[19]$} \\
$\log \varepsilon(\mathrm{N})$ & 8.3 & {$[19]$} \\
$\log \varepsilon(\mathrm{O})$ & 8.4 & {$[19]$} \\
\hline
\end{tabular}

a two-dimensional interpolation made. The scattering function obtained was stored in separate frames, which were then subtracted from the original images. The spectral orders were extracted from the reduced images of the stellar spectrum, flat field, and standard reference spectrum, and the flat-field reduction was carried out. The wavelength scale was calibrated using a two-dimensional polynomial approximation for identified spectral lines.

To study the line-profile variability, the spectra obtained were normalized to the continuum determined for each echelle order. The continuum level in spectral orders with narrow spectral lines was determined using the technique suggested by Shergin et al. [26], which applies smoothing of the spectrum with a variable Gaussian-like filter corresponding to the width of the window (25-30 $\AA$ ) and with the same parameters for the entire sequence of spectra.

To determine the position of the continuum in orders containing broad lines, the broad lines were cut out in specified wavelength intervals for all 30 spectra obtained in 2004 with the $6-\mathrm{m}$ telescope. The continuum position was then determined using a polynomial approximation for all wavelengths of the order, except for the regions of the cut-out broad lines. The parameters of the approximation remained unchanged for all spectra in the series. This procedure provides stable and reproducible determination of the continuum level for all spectra with an accuracy of up to several tenths of a per cent. This enabled us to reach high accuracy in our determination of differential line 
Table 2. Observations of $\rho$ Leo in 2004

\begin{tabular}{c|c|c|c|c|c}
\hline $\begin{array}{c}\text { Date } \\
(2004)\end{array}$ & $\begin{array}{c}\text { Observatory, } \\
\text { telescope }\end{array}$ & $\begin{array}{c}\text { Spectrograph, } \\
\text { detector }(\mathrm{CCD})\end{array}$ & $\begin{array}{c}\text { Number } \\
\text { spectra }\end{array}$ & $\begin{array}{c}\text { Exposure, } \\
\text { min }\end{array}$ & $\begin{array}{c}\text { Total time } \\
\text { observations, } \mathrm{h}\end{array}$ \\
\hline 10-11 January & SAO, 6.0-m & NES, $2048 \times 2048$ pix. & 30 & 6 & 3.5 \\
& BOAO, $1.8-\mathrm{m}$ & BOES, $2048 \times 4096$ pix. & 2 & $9 / 10$ & 0.6 \\
14/15 January & BOAO, 1.8-m & BOES, $2048 \times 4096$ pix. & 11 & 4 & 2.5 \\
3/5 February & BOAO, $1.8-\mathrm{m}$ & BOES, $2048 \times 4096$ pix. & 4 & $4 / 7$ & 3.0 \\
\hline
\end{tabular}

profiles and to detect variability in profiles of broad lines at the $0.2 \%$ level.

Spectral observations of $\rho$ Leo at the BOAO were made on four nights, on January 11, 14, and 15 and February 5, 2004 with the BOES fiber echelle spectrograph [27] mounted on the 1.8-m telescope and outfitted with a CCD detector $(2048 \times 4096$ pixels, with a pixel size of $15 \times 15$ micron).

We obtained 17 echelle spectra with a spectral resolution of $R \approx 44000$ in the broad wavelength interval $3782 \AA<\lambda<9803 \AA$. Since the star is bright, the ratio $S / N \approx 300$ was reached with an exposure time of 4-10 min. The time interval between successive spectra was 6-10 min for each night.

The preliminary reduction of the CCD images of the echelle spectra was carried out in the IRAF package, and the subsequent reduction of the spectra using the modified 2004 version of the DECH package (DECH 20T) [28]. To study the line-profile variability, the spectra were normalized to the continuum level, which was determined using the method described in [16].

The wavelengths for all 47 spectra obtained in 2004 were transformed to a heliocentric scale in the standard way. Further, to make the analysis of the profile variability more convenient, the average radial velocity of the center of mass was used as the zeropoint of the wavelength scale: $V_{\text {rad }}=42 \mathrm{~km} / \mathrm{s}$ [24].

As an example, Fig. 1 illustrates the average spectrum of $\rho$ Leo determined from 30 SAO spectra obtained on January 10-11, 2004, for spectral orders in which the lines analyzed here are present.

\section{PROFILE VARIATIONS}

\section{SEARCH FOR REGULAR VARIABILITY}

\subsection{Variations of Average Line Profiles}

Line profiles in spectra of $\mathrm{O}$ and $\mathrm{B}$ stars averaged during a night often display substantial variability [6]. To illustrate the night-to-night profile variations in the spectrum of $\rho$ Leo, Fig. 2 presents nightly averaged profiles of the NII $\lambda 5676.019$ (NII $\lambda$ 5676) and NII $\lambda 5679.562$ (NII $\lambda$ 5680) lines and the HeI $\lambda$ 5875.621, 5875.966 (HeI $\lambda$ 5876) doublet.

Figure 2 shows that the amplitude of the line-flux variations in the spectrum of $\rho$ Leo is $1-2 \%$ in units of the intensity of the adjacent continuum. The SAO and $\mathrm{BOAO}$ line profiles averaged for the night of Januray 11, 2004 coincide within the errors, demonstrating good intrinsic accuracy of the procedures used to determine the continuum level.

Numerous strong lines of the NII ion stand out, and are particularly prominent at 5660-5715 $\AA$, due to the substantial nitrogen excess of the star compared with the solar abundance (0.5 dex) [29] (Table 1).

\subsection{Variations of the Differential Line Profiles}

We constructed differential profiles (an individual profile minus an average profile) in order to better distinguish variable features in the line profiles. We used two sets of spectra to obtain the average profiles: 30 SAO spectra obtained on January 10-11, 2004 and 17 BOAO spectra obtained in 2004.

To illustrate the results, Fig. 3 presents dynamical differential profiles of the NII $\lambda 5495.666$ (NII $\lambda$ 5496), NII $\lambda$ 5666.667, NII $\lambda$ 5679.562, SiIII $\lambda$ 5695.522 (SiIII $\lambda$ 5696), SiIII $\lambda 5739.733$ (SilII $\lambda$ 5740), and HeI $\lambda 5876$ lines in the spectrum of $\rho$ Leo in terms of the Doppler shifts from the line centers, constructed using the SAO spectra obtained on January 11, 2004. These lines were selected because they were visible in both the SAO and BOAO spectra. In addition, we selected sufficiently strong lines (with residual line intensities $r<0.95$ ), so that line-profile variations due to NRPs could be discriminated against the background of noise variations of the profiles.

The deviations of individual profiles from those averaged over all the spectra are marked by shades of gray. For increased clarity, the differential profiles are presented in "negative," i.e., dark areas correspond to profile sections above the average (profile peaks), and light areas (depressions) to profile sections below the average. Regular profile variations can be seen in the diagrams in Fig. 3. 


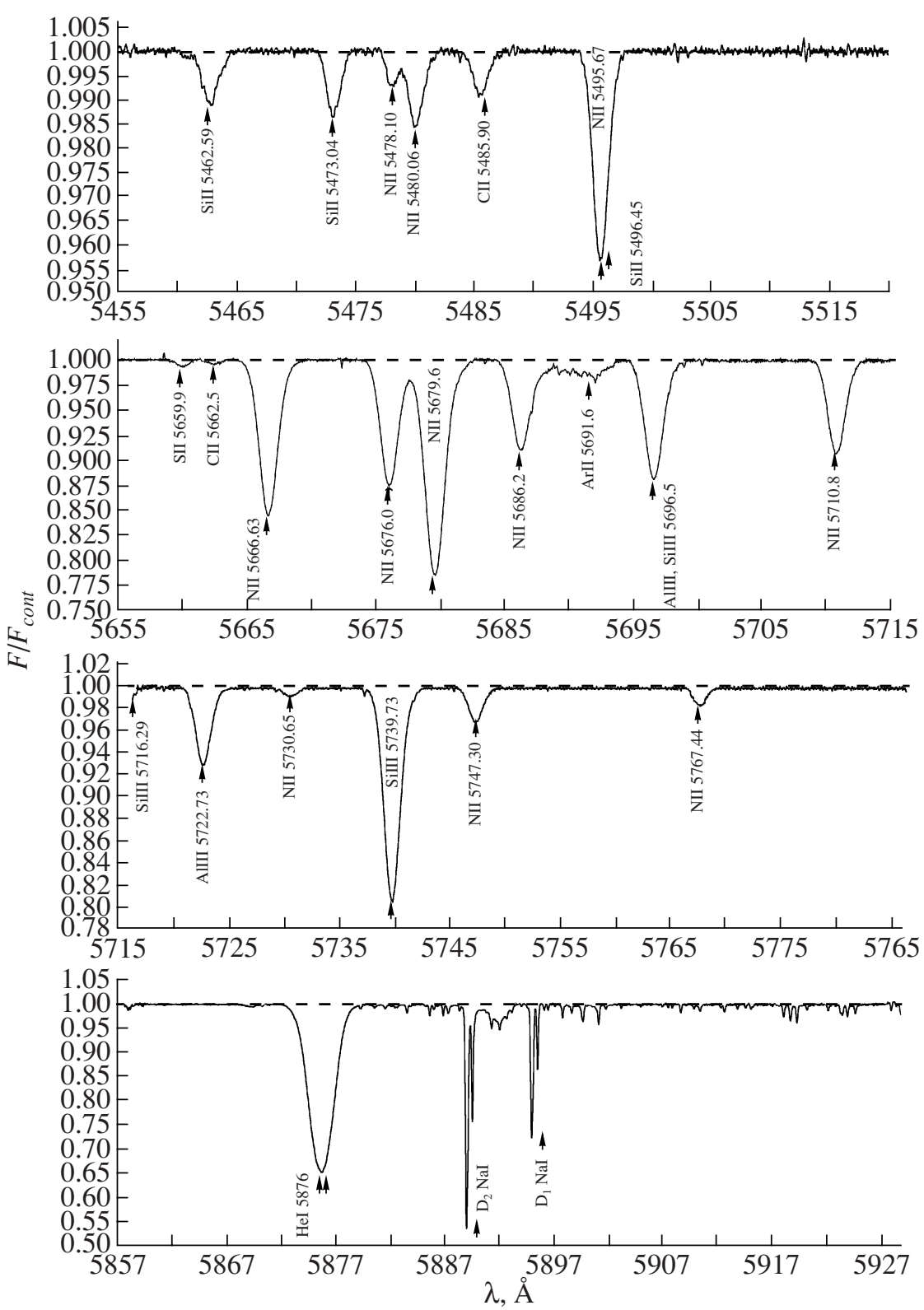

Fig. 1. Average spectra of $\rho$ Leo at $\lambda \lambda 5455-5520 \AA, \lambda \lambda 5655-5715 \AA, \lambda \lambda 5715-5785 \AA$ and $\lambda \lambda 5857-5927 \AA$. The vertical arrows indicate the laboratory wavelengths of the lines.

A broad (50-100 km/s), variable quasiabsorption feature is observed (a lighter region in the dynamical spectrum) in the region of the HeI $\lambda 5876$ line. This feature appears at a velocity of about $-100 \mathrm{~km} / \mathrm{s}$ in the first (lower) differential profiles. With time, it shifts towards the center of the lines. In the last (upper) profiles, this feature is seen at positive velocities. This behavior of the differential profiles is common for NRPs [15, 16, 30]. A similar feature is observed in the dynamical spectra of the profile variations for the NII $\lambda$ 5667, NII $\lambda$ 5680, and SiIII $\lambda 5740$ lines. The naked-eye variations of spectrum-to-spectrum profiles for weaker lines are only weakly pronounced, due to both the short observation time and the small amplitude of the profile variations.

Regular profile variations are evident in the diagrams. We can see that profile peaks (darker regions) shift from the red to the blue wing of a profile. Such profile variations are common for NRPs in the $(l, m)=(2,2)$ quadrupole mode [16].

Similar profile variations are also found in the BOAO spectra. Figure 4 presents dynamical variations of the line profiles in the spectrum of $\rho$ Leo for all observations of the star. These variations are less clearly visible due to the smaller number of spectra 

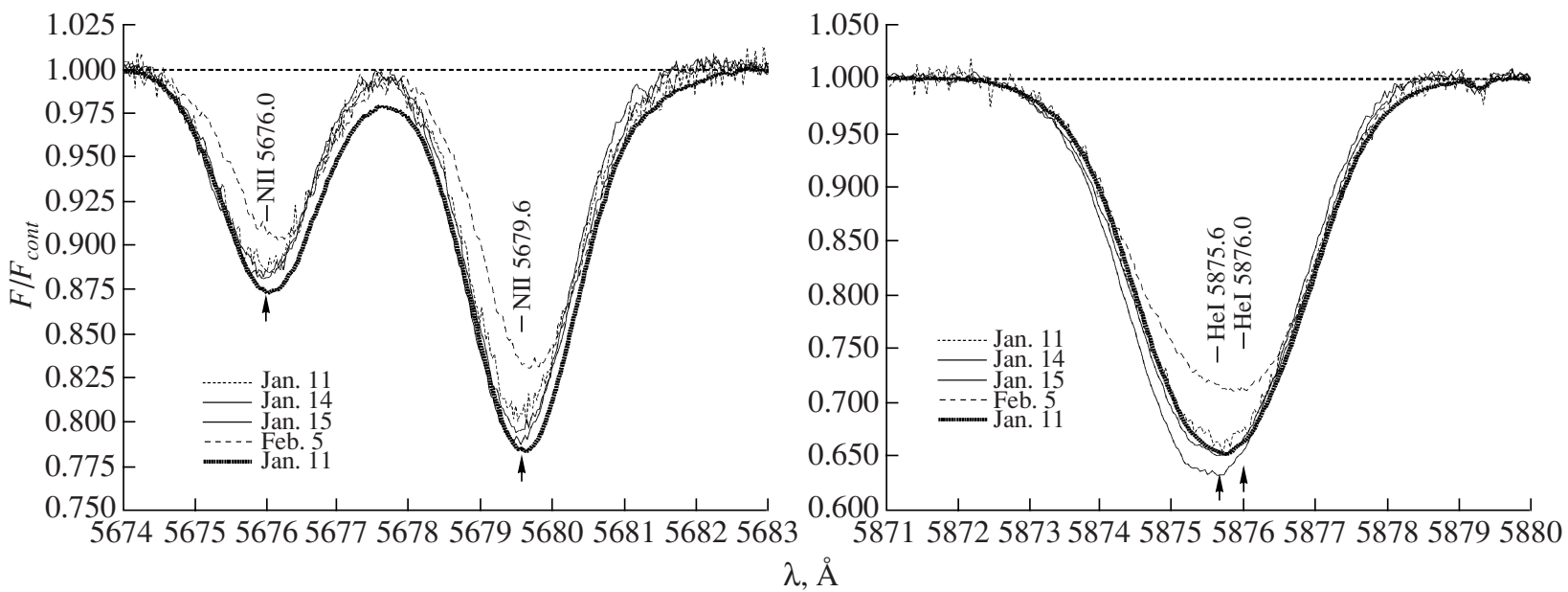

Fig. 2. Average line profiles for the NII $\lambda$ 5676.019, NII $\lambda 5679.562$ lines (left), and the HeI $\lambda \lambda 5875.621,5875.966$ doublet for the BOAO spectra obtained on January 11, 14, 15 and February 5, 2004 and the SAO spectra obtained on January 11, 2004. The vertical arrows indicate the laboratory wavelengths of the lines.
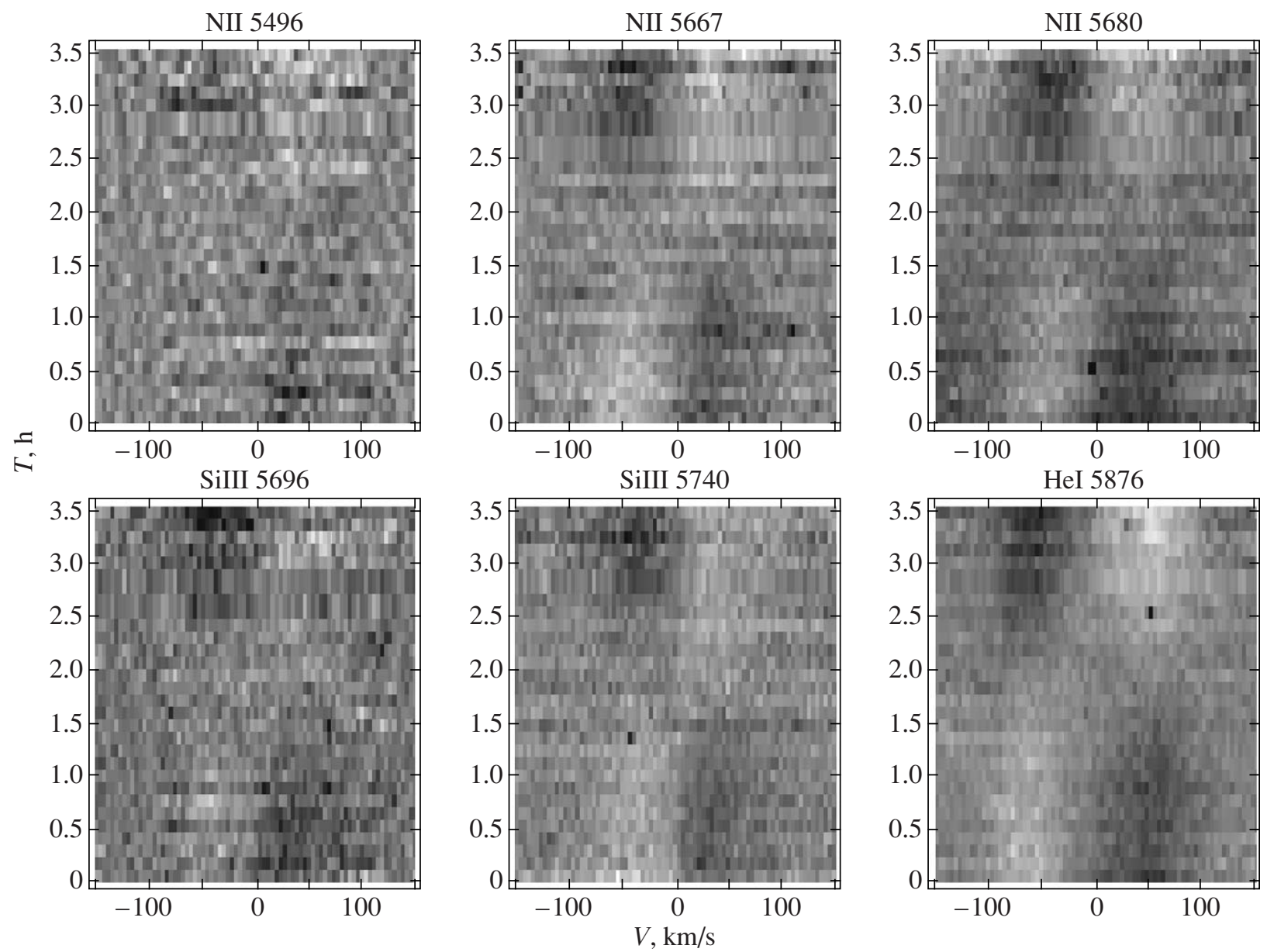

Fig. 3. Dynamical spectra of line-profile variations for the NII $\lambda$ 5495.67, NII $\lambda$ 5666.63, NII $\lambda$ 5679.56, SiIIII $\lambda$ 5695.52, SiIII $\lambda$ 5739.73, and HeI $\lambda 5876$ lines in the spectrum of $\rho$ Leo in terms of the Doppler shift from the center of the lines (horizontal axis) for the SAO observations on January 10-11, 2004. Deviations of individual line profiles from the average are indicated by the gray shades. Light and dark areas correspond to regions of the profiles below and above the average level, respectively. The interval between successive spectra was 7 min. 


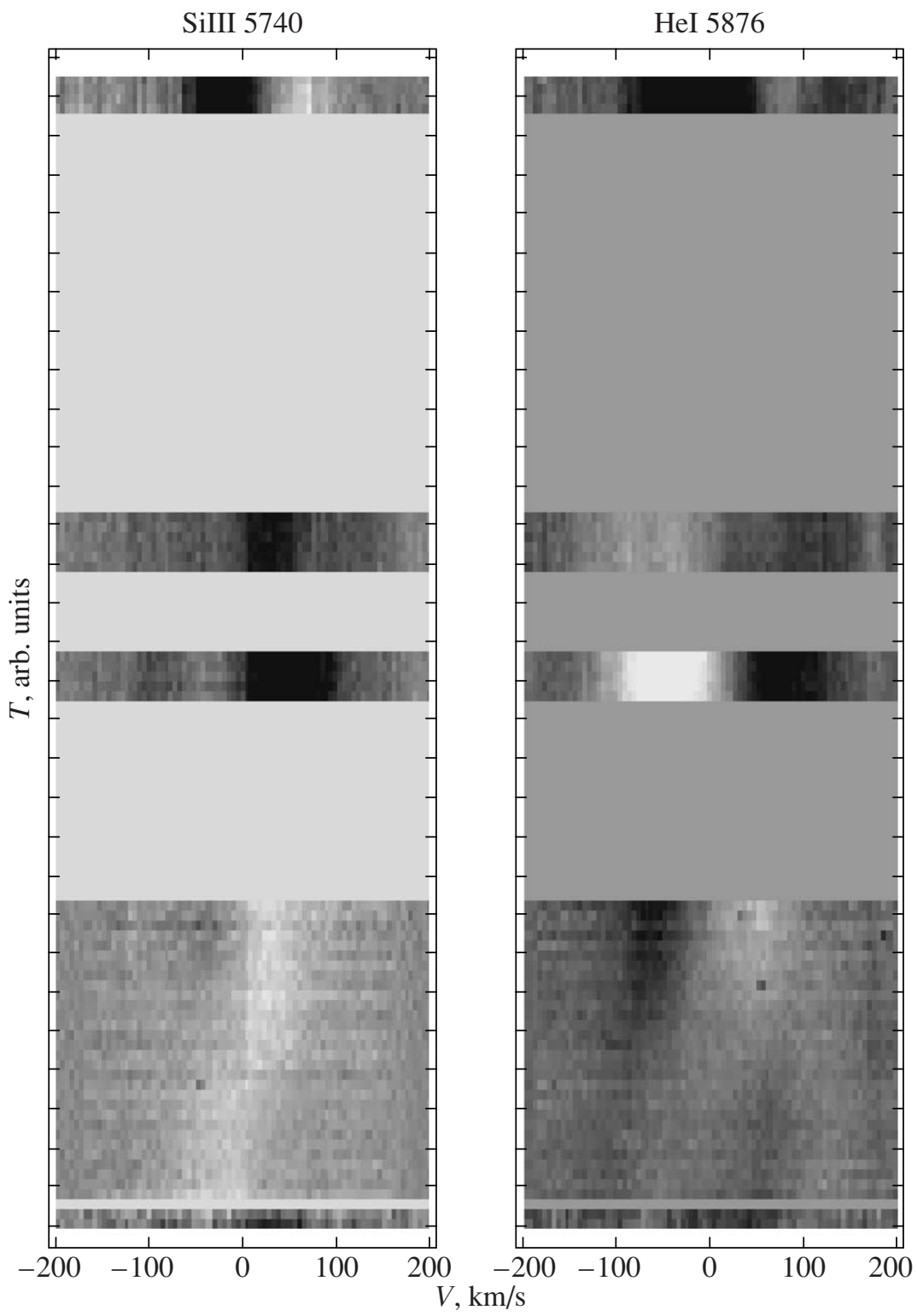

Fig. 4. Same as Fig. 3 for dynamical spectra of the profile variations of the SiIII $\lambda 5739.73$ and HeI $\lambda 5876$ lines for the total observation time. The intervals between observations made on different nights are shown by areas of gray (not to scale).

obtained with the 1.8-m BOAO telescope and the larger intervals between the spectra obtained on different nights.

\subsection{Search for Regular Line-Profile Variations}

We searched for regular components of the lineprofile variations in the spectrum of $\rho$ Leo via a Fourier analysis of the profile variability. The Fourier spectra were cleaned from false peaks using a modification of the CLEAN procedure [31] described in [32, 33]. For each of the studied lines, we constructed a time series of $\Delta F(t, V)$-the differential fluxes for a specified profile at time $t$ for a Doppler shift from the profile center in the center-of-mass frame $V$.
The CLEAN procedure was applied for all $V$ values obtained in the profiles of the studied lines. The Fourier analysis of the line-profile variations was carried out in two stages. In the first, the SAO spectra obtained on January 10-11, 2004 were analyzed, and in the second, all spectra obtained in 2004.

\subsubsection{Fourier analysis of SAO observations.} Figure 5 presents for all the studied lines plots of the squared amplitude of the Fourier transform (Fourier power spectra) constructed using only $30 \mathrm{SAO}$ spectra. To make the power spectra (periodogram) clearer, all values corresponding to small significance levels $q<10^{-3}$ for the presence of a strong white-noise peak in the periodogram were rejected. Thus, the 

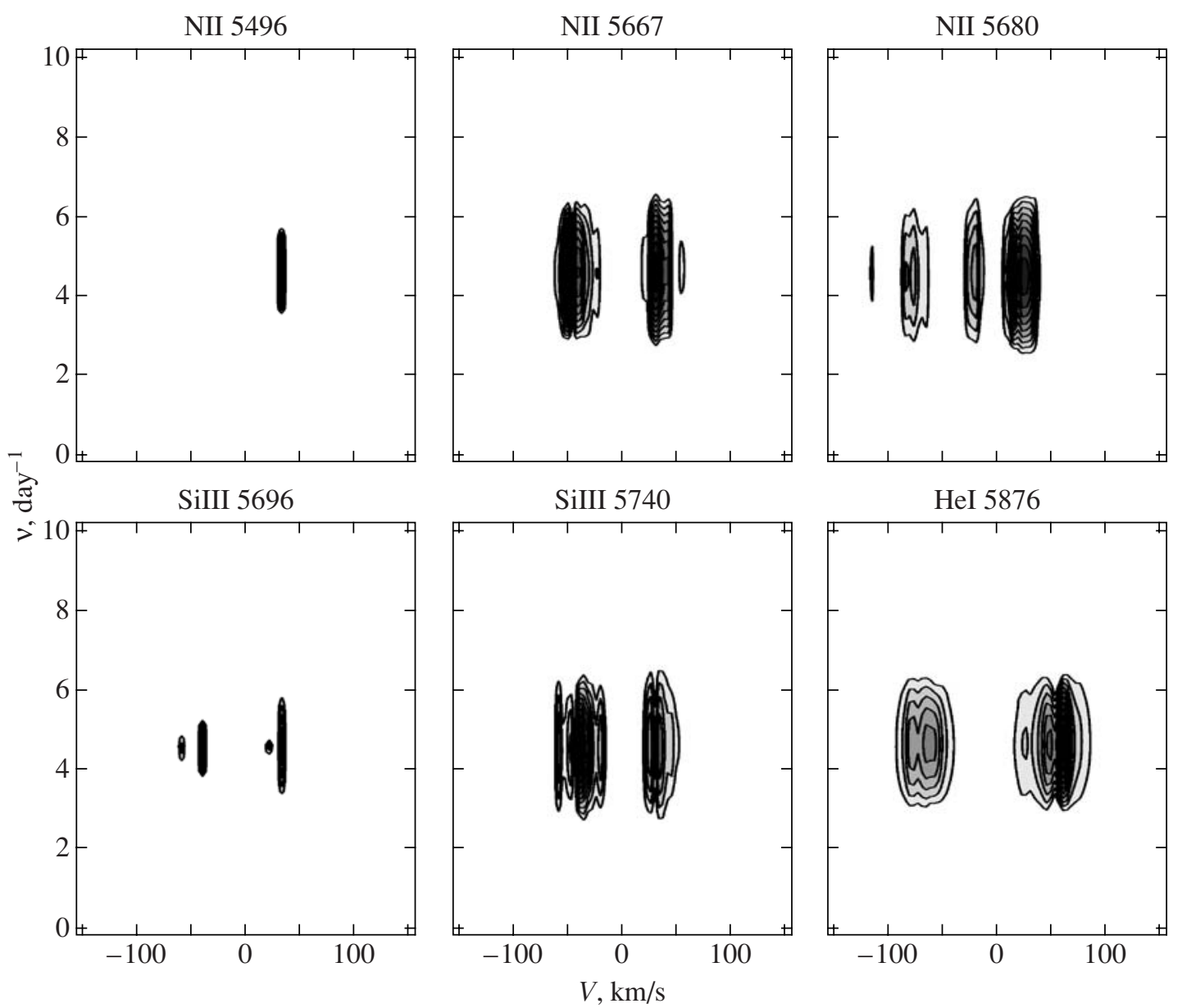

Fig. 5. Fourier-spectra of variations of the differential profiles for the NII $\lambda$ 5495.67, NII $\lambda$ 5666.63, NII $\lambda$ 5679.56, SiIII $\lambda$ 5695.52, SiIII $\lambda$ 5739.73, and HeI $\lambda 5876$ lines in the spectrum of $\rho$ Leo at frequencies $\nu=0-10$ day ${ }^{-1}$ derived from the SAO observations of January 10-11, $2004 \AA$. Only amplitudes of the Fourier spectra corresponding to significance levels $q<10^{-3}$ for the presence of a strong peak in the white noise periodogram are given. Darker regions correspond to larger amplitudes.

graphs present only significant components of the periodogram.

Figure 5 shows a broad peak at $\nu \approx 4.7$ day $^{-1}$. The large width of the peak is due to the relatively low resolution of the Fourier spectrum because of the short duration of the observations $T$. The accuracy of the frequency of the harmonic component is $\Delta \nu \approx$ $1 / T$, while $P=1 / \nu \approx 5.1^{h}$ exceeds the length of the observation time, and so cannot be directly identified with a period of regular variations of the profiles. We will call this the quasi-period of the regular profile variations.

\subsubsection{Fourier analysis of all the observations.} When constructing the complete Fourier spectrum for the profile variations, we also included 17 observations made at the BOAO. The total duration of all the observations is 24.8 days, which yields $\Delta \nu=$ $1 / T \approx 0.04$ day $^{-1}$. Our estimates show that the real accuracy of the period is lower: $\approx 0.1$ day $^{-1}$, due to the large interval of $\approx 21$ day between the main set of observations and the last four BOAO spectra obtained on February 5, 2004.

Figure 6 presents plots of the squared amplitude of the Fourier transform for all the studied NII, SiIII, and HeI lines constructed using all 47 spectra obtained in 2004 at both the SAO and BOAO. Due to the large gaps in the time series, we used the CLEAN procedure to remove false peaks. A threshold significance level of $q=10^{-3}$ for the presence of a strong whitenoise peak in the periodogram was applied.

Several dozen individual peaks can be seen in the calculated periodograms. However, components significant at the level $q<10^{-3}$ are found only at frequencies $\nu=0.1-6.5$ day $^{-1}$. Table 3 presents the frequencies of the harmonic components of the Fouriertransform power spectrum visible in the profile varia- 


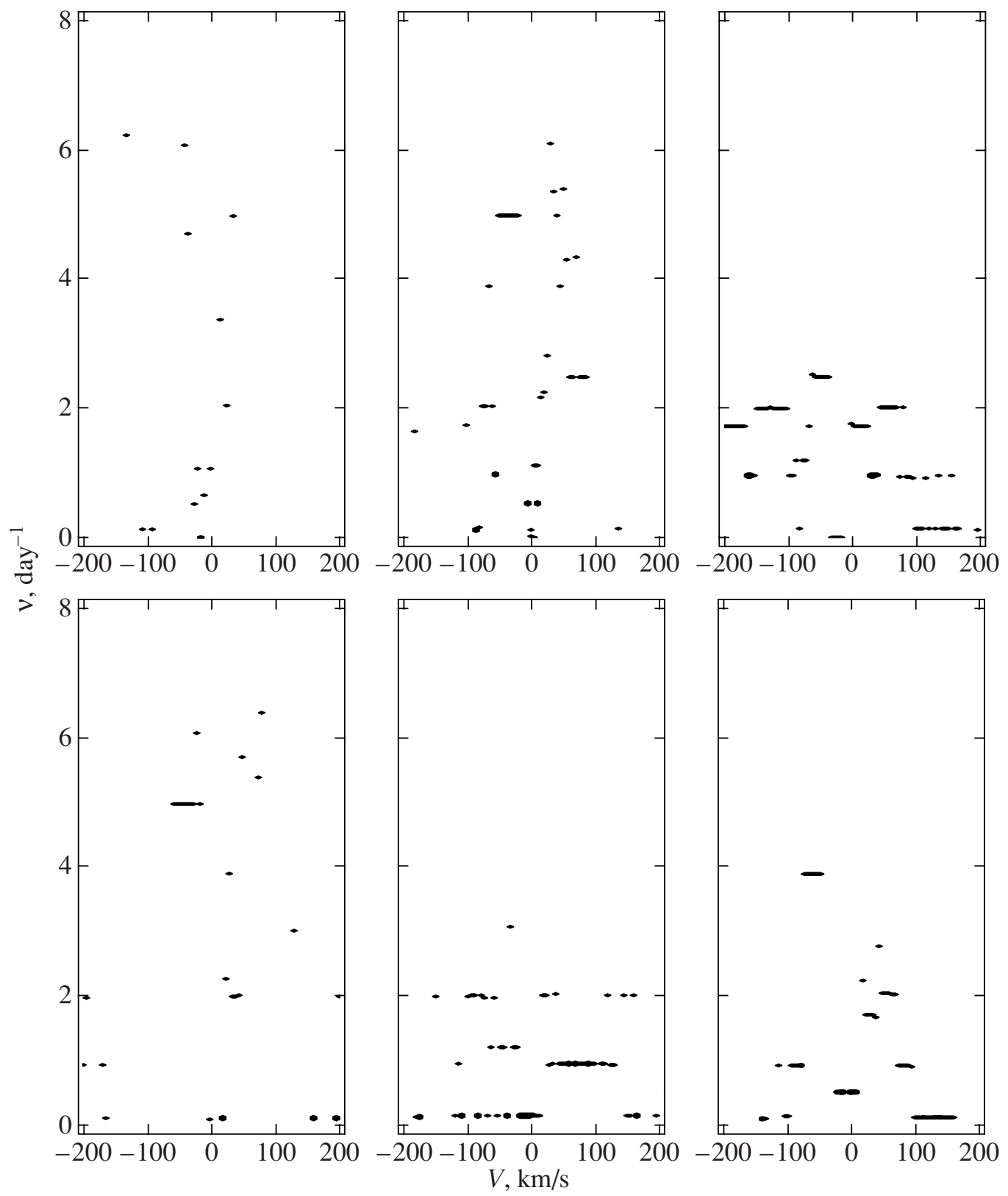

Fig. 6. Same as Fig. 5 for frequencies $\nu=0-8$ day $^{-1}$ derived from the SAO and BOAO observations of January-February 2004.

tions for no fewer than two lines in the spectrum of $\rho$ Leo.

\section{DISCUSSION OF THE RESULTS}

Our analysis indicates the possible presence of regular line-profile variations in the spectrum of the $\rho$ Leo system with periods from $\approx 7 \mathrm{~d}$ to $P=4-6 \mathrm{~h}$. Such a large range of periods suggests that the detected profile variations have different natures. The data in Table 3 for the derived frequencies of the regular profile variations can be divided into three groups: the first with the frequency $\nu_{1} \approx 0.14$ day $^{-1}$, the second with the frequencies $\nu_{2}-\nu_{5}$, and the third with the frequencies $\nu_{7}, \nu_{8}$, and $\nu_{10}$.

The frequency $\nu_{1}$ probably corresponds to the period of rotation of the star, $P_{1}=7^{\mathrm{d}} \pm 2^{\mathrm{d}}$. Adopting $R_{*} \approx 35 R_{\odot}$, the average radius of $\rho$ Leo from Table 1 , and $V \sin i=75 \mathrm{~km} / \mathrm{s}$ (Table 1), we find the upper limit for the rotational period of the star $P_{\text {rot }} \leq 47^{\mathrm{d}}$. Since $P_{1}$ substantially exceeds the possible pulsation periods B1 stars [20], $P_{1}$ can probably be identified with the rotational period of the star $P_{\text {rot }}$. 
Table 3. Frequencies $(\nu)$ and corresponding periods $(P)$ for harmonics of the line-profile variations in the spectrum of $\rho$ Leo. The presence of a given frequency component in the periodogram is marked with a plus sign. A bold plus in the last column means that the component with the given frequency seems to be real; a colon indicates that the given harmonic may belong to a false peak of the periodogram

\begin{tabular}{r|c|c|c|c|c|c|c|c|c}
\hline No. & $\nu$, day $^{-1}$ & $P$ & $\begin{array}{c}\text { NII } \\
\lambda 5496\end{array}$ & $\begin{array}{c}\text { NII } \\
\lambda 5667\end{array}$ & $\begin{array}{c}\text { NII } \\
\lambda 5680\end{array}$ & $\begin{array}{c}\text { SiII } \\
\lambda 5696\end{array}$ & $\begin{array}{c}\text { SiII } \\
\lambda 5740\end{array}$ & $\begin{array}{c}\text { HeI } \\
\lambda 5876\end{array}$ & State \\
\hline 1 & $0.14 \pm 0.1$ & $7^{\mathrm{d}} \pm 2^{\mathrm{d}}$ & + & + & + & + & + & + & + \\
2 & $0.60 \pm 0.1$ & $1.8^{\mathrm{d}} \pm 0.3^{\mathrm{d}}$ & + & + & - & - & + & + & + \\
3 & $0.94 \pm 0.1$ & $1.1^{\mathrm{d}} \pm 0.1^{\mathrm{d}}$ & - & - & + & + & + & + & + \\
4 & $1.15 \pm 0.1$ & $21^{\mathrm{h}} \pm 2^{\mathrm{h}}$ & - & + & + & - & + & + & + \\
5 & $1.74 \pm 0.1$ & $14^{\mathrm{h}} \pm 1^{\mathrm{h}}$ & + & + & + & - & - & + & + \\
6 & $2.03 \pm 0.1$ & $11.8^{\mathrm{h}} \pm 0.5^{\mathrm{h}}$ & + & + & + & + & + & + & $:$ \\
7 & $3.9 \pm 0.1$ & $6.1^{\mathrm{h}} \pm 0.2^{\mathrm{h}}$ & + & + & - & + & - & + & + \\
8 & $4.4 \pm 0.1$ & $5.3^{\mathrm{h}} \pm 0.1^{\mathrm{h}}$ & - & + & - & + & - & + & + \\
9 & $5.0 \pm 0.1$ & $4.8^{\mathrm{h}} \pm 0.1^{\mathrm{h}}$ & + & + & - & + & - & - & $:$ \\
10 & $6.2 \pm 0.1$ & $3.8^{\mathrm{h}} \pm 0.1^{\mathrm{h}}$ & + & - & - & + & - & - & + \\
\hline
\end{tabular}

We tentatively conclude that either $P_{1}$ is equal to the rotational period or the rotational period is a multiple of $P_{1}$, if $\nu_{1}$ is an overtone of the rotational frequency of the star. If $\nu_{1}$ is the first overtone of the rotational frequency, then $P_{\text {rot }} \approx 14^{\mathrm{d}}$, if $\nu_{1}$ is the second overtone, $P_{\text {rot }} \approx 21^{\mathrm{d}}$, and so on. Rotational periods from $\approx 3^{\mathrm{d}}-4^{\mathrm{d}}$ to $\approx 20^{\mathrm{d}}$ are common for late$\mathrm{O}$ and early-B stars $[6,8,17]$.

Let us now consider the second group of frequencies, $\nu_{2}-\nu_{5}$, which correspond to periods of $0.6-$ 1.8 days. We note first of all that, within the uncertainties of the frequencies of the harmonic components, $\nu_{4} \approx 2 \nu_{2}$ and $\nu_{5} \approx 2 \nu_{3}$; therefore, the components $\nu_{4}$ and $\nu_{5}$ may be overtones of the frequencies $\nu_{2}$ and $\nu_{3}$, respectively. We will accordingly concentrate on analyzing the frequencies $\nu_{2}$ and $\nu_{3}$.

One possible origin of rapid line-profile variations with periods of $1-2$ days is rotational modulation [1, $2]$. The model [1] supposes that the expanding atmospheres of hot, early-type stars contain corotational jets that rotate with the angular velocity of the star. Additional absorption of the star's radiation due to the passage of these jets through the line of sight results in regular variations of the line profiles. The period of such variations will be $P_{n}=P_{\text {rot }} / n$, where $n$ is the number of corotational jets. The periods of the profile variations $P_{2}=1.8^{\mathrm{d}}$ and $P_{3}=1.1^{\mathrm{d}}$ correspond to the values $n=4$ and $n=6$, which seems fundamentally possible if the rotational period of the star is $P_{\text {rot }} \approx 7^{\mathrm{d}}$.
At the same time, we cannot rule out a priori the possibility that the profile variations with frequencies from the second group are pulsational. To test this hypothesis, we plot the frequencies of the second group of profile variations in a $T_{e f f}-P$ diagram (Fig. 7), where $P$ is the period of the quadrupole $(l=2)$ pulsations of hot main-sequence stars [20, Fig. 6]. With $T_{\text {eff }}=24200 \mathrm{~K}$, the obtained frequencies are outside the zone of pulsational instability for OB stars, making it very unlikely that pulsations of the star are the origin of the profile variations with the indicated frequencies for the given temperature. With $T_{\text {eff }}=20260 \mathrm{~K}$, the derived frequencies will fall in the region of high $g$-modes of quadrupole pulsations. At the same time, these frequencies belong to mainsequence stars with masses $M \leq 10 M_{\odot}$, which are appreciably lower than the value presented in [17].

The third group of regular frequencies of the profile variations $\nu_{7}, \nu_{8}$, and $\nu_{10}$ corresponds to periods $P$ from $\approx 4^{\mathrm{h}}$ to $\approx 6^{\mathrm{h}}$. Such values for $P$ are common for profile variations associated with NRPs in OB stars [5].

The $(l, m)$ NRP mode can be specified from the relation $l \approx 0.1+1.09\left|\Delta \varphi_{0}\right| / \pi$ and $m \approx 1.33+$ $0.54\left|\Delta \phi_{1}\right| / 2 \pi$, where $\Delta \varphi_{0}=\varphi_{\text {red }}-\varphi_{\text {blue }}$ is the phase difference of the Fourier components of the profile variations for the red $\left(\varphi_{\text {red }}\right)$ and blue $\left(\varphi_{\text {blue }}\right)$ wings of the lines of the main NRP component $\nu_{0}$. $\Delta \varphi_{1}$ is specified analogously for the first harmonic of the line-profile variations $\nu_{1}=2 \nu_{0}$ [34]. 


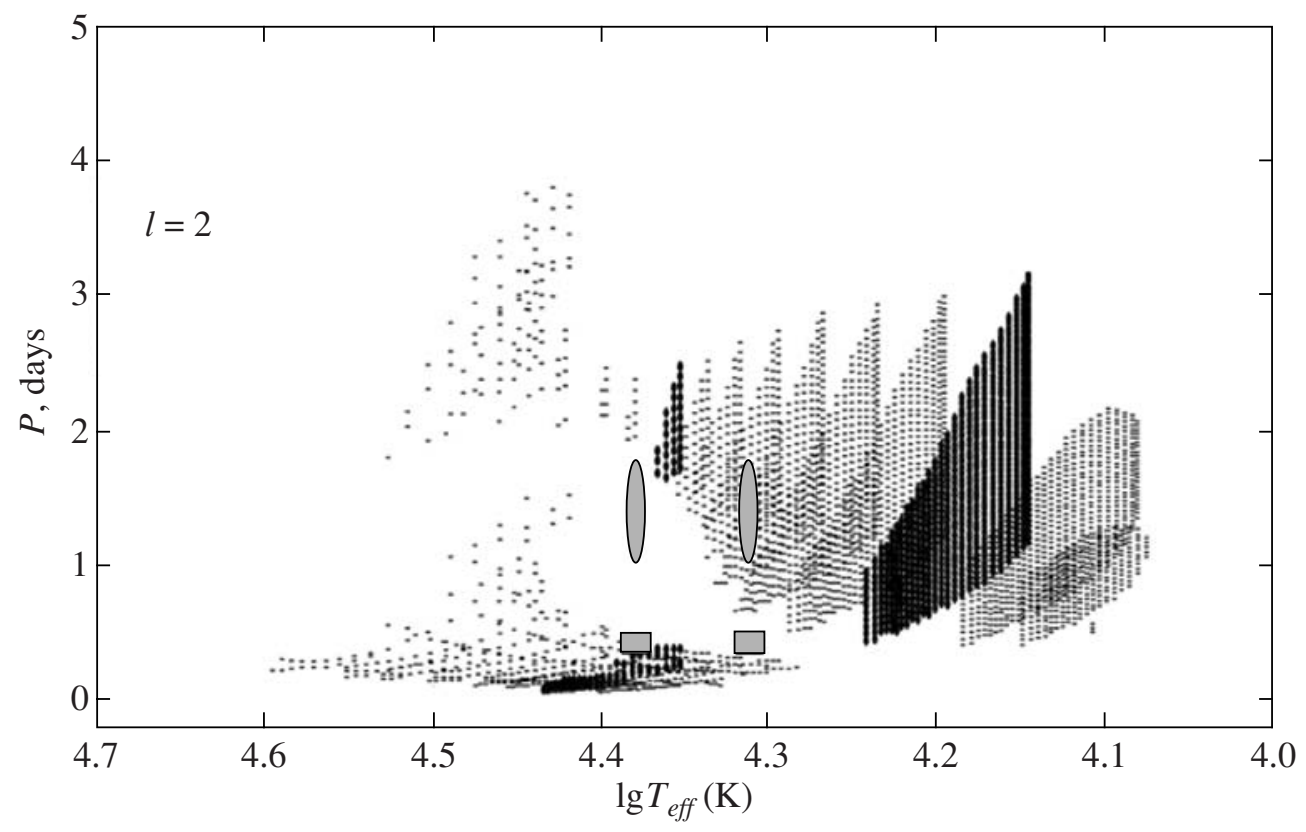

Fig. 7. Pulsation periods of $\beta$ Cep stars and slowly pulsating B (SPB) stars in the region of effective temperatures $10^{4}-5 \times$ $10^{4} \mathrm{~K}$ (points), according to the calculations of [20]. The upper region of periods belongs to $g$ pulsation modes of SPB stars, and the lower to pulsations of massive $\beta$ Cep stars in the quadrupole mode $(l=2)$. The gray rectangles and ellipses indicate the frequencies of regular profile variations in the spectrum of $\rho$ Leo. Ellipses indicate the frequency range $0.60-0.94 \mathrm{~d}^{-1}$ (periods $P=1.06-1.67 \mathrm{~d}$ ) and rectangles the frequency range $3.9-6.2 \mathrm{~d}^{-1}$ (periods $P=0.16-0.26 \mathrm{~d}$ ) for regular components of the profile variations in the spectrum of $\rho$ Leo. The left rectangle and ellipse correspond to $T_{\text {eff }}=24200 \mathrm{~K}$, and the right to $T_{\text {eff }}=20260 \mathrm{~K}$.

Unfortunately, due to the short duration of our data sequence, $\Delta \varphi_{0}$ and $\Delta \varphi_{1}$ cannot be determined very accurately. For the profile of the HeI $\lambda 5876$ line, we can determine only that $\left|\Delta \varphi_{0}\right|$ is in the velocity intervals -80 to $-50 \mathrm{~km} / \mathrm{s}$ and -100 to $-50 \mathrm{~km} / \mathrm{s}$. Extrapolating the phase difference in these intervals to the total range of velocities $\pm V \sin i$ yields $l \geq 2$.

To determine to what extent the derived periods for the line-profile variations in the spectrum of $\rho$ Leo correspond to possible pulsation periods of OB stars, we plot the frequencies of the third group of components in a $T_{\text {eff }}-P$ diagram. The positions of harmonic components for $T_{\text {eff }}=24200 \mathrm{~K}$, presented in Fig. 7 , are located in the region of pulsational instability of $\beta$ Cep stars. At the same time, the positions of the same components for $T_{e f f}=20260 \mathrm{~K}$ lie outside the region of pulsations of OB stars (Fig. 7).

Thus, one possible explanation for the nature of the profile variations in the spectrum of $\rho$ Leo is that $T_{\text {eff }}$ is close to $24200 \mathrm{~K}$; in this case, profile variations with frequencies from the third group $\left(4-6 \mathrm{~d}^{-1}\right)$ are associated with NRPs of the star in the quadrupole mode. Slower variations with frequencies from the second group most likely occur due to rotational modulation of the profiles.
However, this interpretation is not the only one possible, since the theory developed for mainsequence stars likely cannot be directly applied to OB supergiants. In addition, we cannot rule out the possibility that $\rho$ Leo pulsates in higher modes than the $l=2$ quadrupole mode. We plan more extensive observations to more reliably discriminate between profile variations in the spectrum of $\rho$ Leo due to rotational modulation and NRPs, and also to determine the rotational period of the star.

The presence of a magnetic field results in a substantially different pattern of line-profile variability [13]. One consequence of the presence of a magnetic field in the star is the formation of quasi-regular structures (corotational jets) in its atmosphere [1, 2]. Thus far, searches for a magnetic field in $\rho$ Leo have been unsuccessful [35]. On the other hand, our analysis of the line-profile variations indicates that the regular profile variations extend beyond the range $\pm V \sin i= \pm 60 \mathrm{~km} / \mathrm{s}$ (Figs. 3, 6). In the presence of a magnetic field, photospheric NRPs can lead to regular fluctuations of the density of the stellar wind, which will result in regular profile variability beyond $\pm V \sin i[36]$.

Our estimates show that, out to $\approx 2 R_{*}$, where $R_{*}$ is the radius of the star, the magnetic pressure $B^{2} / 8 \pi$ 
will exceed the gas pressure for moderate $B \approx 100 \mathrm{G}$. In this case, regular profile variability would be seen to $\pm 2 V \sin i$, in agreement with the observed pattern of the profile variability. Note also that the $V \sin i$ value in Table 2 may be underestimated. In a future study, we will analyze polarization observations of $\rho$ Leo made with the SAO 6-m telescope in 2005-2006, enabling us to estimate the strength of the magnetic field of the star.

\section{CONCLUSIONS}

We can draw the following conclusions from our observations of $\rho$ Leo and our analysis of profile variations for the NII $\lambda$ 5495.67, NII $\lambda$ 5666.63, NII $\lambda$ 5679.56, SiIII $\lambda$ 5696, SiII $\lambda$ 5739.73, and HeI $\lambda 5876$ lines.

(1) All the studied lines display variable profiles, with amplitudes of $0.5-1 \%$.

(2) The line-profile variations can include regular components with characteristic time scales from $\approx 3^{\mathrm{d}}$ to $6^{\mathrm{h}}$.

(3) A component with the period $\approx 7^{\mathrm{d}} \pm 2^{\mathrm{d}}$ is probably related to the star's rotation, while regular components with periods $P=0.6^{\mathrm{d}}-1.8^{\mathrm{d}}$ may be due to rotational modulation of the line profiles.

(4) We have detected short-period variations of the line profiles with the characteristic time scales $3.8^{\mathrm{h}}-6.1^{\mathrm{h}}$. We suggest that these components correspond to non-radial pulsations of the star with multipolarities $l \geq 2$.

\section{ACKNOWLEDGMENTS}

This work was supported by the Russian Foundation for Basic Research (project no. 05-02-16995a), the Program of Support of Leading Scientific Schools of the Russian Federation (grant no. NSh8542.2006.2), and a Presidential Grant of the Russian Federation for the Support of Young Russian PhDs in Science (grant no. MK-874.2004.2). G.G.V. is grateful to the Ministry of Science and Technology of South Korea (Korea MOST), which supported him with grant M1-0222-00-0005, and the South Korean Science and Technology Society (KOFST) for financial support in the framework of KASI (the Brain Pool Program).

\section{REFERENCES}

1. L. Kaper, H. F. Henrichs, A. W. Fullerton, H. Ando, et al., Astron. Astrophys. 327, 281 (1997).

2. L. Kaper, H. F. Henrichs, J. S. Nichols, J. H. Telting, et al., Astron. Astrophys. 344, 231 (1999).

3. A. Kaufer, O. Stahl, B. Wolf, et al., Astron. Astrophys. 305,887 (1996).
4. S. Lépine, A. F. J. Moffat, Astrophys. J. 514, 909 (1999).

5. J. A. de Jong, H. F. Henrichs, S. Schrijvers, et al., Astron. Astrophys. 345, 172 (1999).

6. J. A. de Jong, H. F. Henrichs, L. Kaper, et al., Astron. Astrophys. 368, 601 (2001).

7. S. M. Kahn, M. A. Leutenegger, J. Cottam, et al., Astron. Astrophys. 365, 365 (2001).

8. N. A. Miller, J. P. Cassinelli, J. J. MacFarlane, D. H. Cohen, Astroph. J. 499, L195 (2002).

9. A. F. Kholtygin, D. N. Monin, A. E. Surkov, and S. N. Fabrika, Pis'ma Astron. Zh. 29, 208 (2003) [Astron. Lett. 29, 175 (2003)].

10. J.-F. Donati, G. A. Wade, J. Babel, et al., Mon. Not. R. Astron. Soc. 326, 1265 (2001).

11. J.-F. Donati, J. Babel, T. J. Harries, et al., Mon. Not. R. Astron. Soc. 333, 55 (2002).

12. J.-F. Donati, I. D. Howarth, J.-C. Bouret, et al., Mon. Not. R. Astron. Soc. 365, L6 (2006).

13. C. Neiner, A. M. Hubert, A. M. Floquet, et al., Astron. Astrophys. 388, 899 (2002).

14. A. F. Kholtygin, J. Brown, S. Fabrika, and A. Surkov, in Magnetic Stars, Ed. by Yu. V. Glagolevskij, D. O. Kydryavtsev, and I. I. Romanyuk (SAO RAN, 2004), p. 250.

15. A. F. Kholtygin, G. Galazutdinov, T. E. Burlakov, et al., Astron. Zh. 83, 252 (2006) [Astron. Rep. 50, $220(2006)]$.

16. A. F. Kholtygin, T. E. Burlakova, S. N. Fabrika, et al., Astron. Rep. 50, 887 (2006).

17. T. Morel, S. V. Marchenko, A. K. Pati, et al., Mon. Not. R. Astron. Soc. 351, 552 (2004).

18. D. R. Gies and D. L. Lambert, Astrophys. J. 387, 673 (1992).

19. P. A. Crowther, D. J. Lennon, and N. R. Walborn, Astron. Astrophys. 446, 279 (2006).

20. A. A. Pamyatnykh, Acta Astron. 49, 189 (1999).

21. V. E. Panchuk, N. E. Piskunov, V. G. Klochkova, et al., Preprint No. 169, SAO RAN (Special Astrophysical Observatory, Russian Academy of Sciences, Nizhniĭ Arkhyz, 2002).

22. V. E. Panchuk, V. G. Klochkova, and I. D. Naidenov, Preprint No. 179, SAO RAN (Special Astrophysical Observatory, Russian Academy of Sciences, Nizhniı̌ Arkhyz, 2003).

23. I. D. Howarth, K. W. Siebert, G. A.J. Hussain, and R. K. Prinja, Mon. Not. R. Astron. Soc. 284, 265 (1997).

24. SIMBAD Astronomical Database (Strasbourg Astronomical Data Center), http://simbad.usrrasbg.fr/Simbad/.

25. P. Ballester, Astron. Astrophys. 286, 1011 (1994).

26. V. S. Shergin, A. Yu. Kniazev, and V. A. Lipovetsky, Astron. Nachr. 317, 95 (1996).

27. Bohyunsan Echelle Spectrograph (Bohyunsan Optical Astronomy Observatory, Korea), http://www. boao.re.kr/BOES/BOESppt3.files/frame.htm.

28. G. A. Galazutdinov, Preprint No. 92, SAO RAN (Special Astrophysical Observatory, Russian Academy of Sciences, Nizhniı̆ Arkhyz, 1992).

ASTRONOMY REPORTS Vol.51 No.11 2007 
29. M. Asplund, N. Grevesse, and A. J. Sauval, in Cosmic Abundances as Records of Stellar Evolution and Nucleosynthesis, Ed. by F. Bash and T. Barnes (San Francisco: Astron. Soc. Pac.), Astron. Soc. Pac. Conf. Ser. 336, p. 25; astro/ph/0410214.

30. Th. Rivinius, D. Baade, and S. Stefl, Astron. Astrophys. 411,229 (2003).

31. D. H. Roberts, J. Lehar, and J. W. Dreher, Astron. J. 93, 968 (1987).

32. V. V. Vityazev, Analysis of Irregular Dynamic Series (St. Pterburg. Gos. Univ., St. Petersburg, 2001) [in Russian].
33. A. F. Kholtygin and A. B. Shneiwais, Astrofizika 48, 87 (2005) [Astrophys. 48, 68 (2005)].

34. J. H. Telting and C. Schrijvers, Astron. Astrophys., Suppl. Ser. 317, 723 (1997).

35. V. D. Bychkov, L. V. Bychkova, and J. Madej, Astron. Astrophys. 407, 631B (2003).

36. S. P. Owocki and S. R. Cranmer, in Radial and Nonradial Pulsations as Probes of Stellar Physics, Ed. by C. Aerts, T. R. Bedding, and J. ChristensenDalsgaard, Astron. Soc. Pac. Conf. Ser. 259, 512 (1988).

Translated by K. Maslennikov 